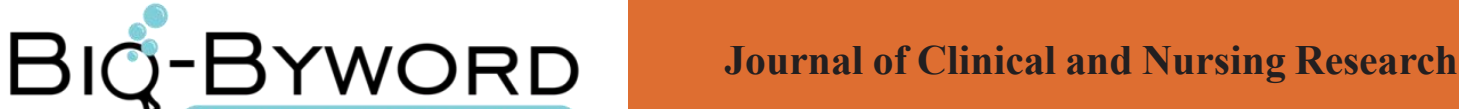

\section{Cause Analysis and Countermeasure of Needle Injury in Surgical Nurs- ing Staff}

Chen Xuejiao

New Hospital of Xinyi City, Jiangsu Province, Xinyi 221400

\section{ARTICLE INFO}

\section{Article history:}

Published online: $30^{\text {th }}$ Sept, 2017

\section{Key words:}

Surgical care

Acupuncture injury

Countermeasure research

\begin{abstract}
Objective: To analyze the causes of needle injury in surgical care workers and provide countermeasures. Methods: A total number of 140 employees were enrolled in this study, and the questionnaire survey and interviews were conducted. The causes of needle injury and the post-injury treatment were observed statistically. Results: A number of 87 nursing staff were stabbed, accounting for $62.1 \%$ of the total number, and $51.7 \%$ of the total number of injuries were injured. Conclusion: In order to avoid the occurrence of needle injury, surgical nurses in the work should pay attention to safety precautions and be promptly after the injury to deal with the wound.
\end{abstract}

\section{Introduction}

In the various departments of the hospital, the surgical workload is large. Surgical care staff at work, not only to deal with busy work, but also at work at any time to face all kinds of danger. Needle injury is the current surgical care, the most common trauma. Medical tools on the human body caused by scratches, stab wounds are within the scope of needle injury, especially surgical care workers often contact the needle, scalpel and other tools, which will cause the deep injury of the skin. Surgical care workers are also very easy to become a high risk of blood transmission population ${ }^{[1]}$. Therefore, our hospital is the surgical care workers in the work of the reasons for needle injury analysis and to explore the corresponding treatment and preventive measures. The investigation report is as follows.

\section{Information and methods}

\subsection{General information}

The number of surgical nurses aged between 25 and 48 years, 65 male nursing staff, 75 female patients, weight $46.5-78.8 \mathrm{~kg}$, the average $(50.12 \pm 1.1) \mathrm{kg}$, working hours are more than one year. Staff education, college education 30 , undergraduate education 60 , undergraduate or higher
50. Staff titles, 50 nurses, 46 nursery care personal, 30 deputy director of the nurse and 14 owner nurses.

\subsection{Survey method}

Using the hospital-made surgical care staff needle injury situation questionnaire, first of 140 employees to conduct an anonymous investigation. After the completion of the investigation record, record the analysis data, interview the staff, the investigation will cause the specific situation of needle injury, and nursing staff in the treatment of needle after the investigation of the actual surgical care staff of the actual situation. To facilitate the investigation of the meeting combined with the actual work of the situation to prevent the treatment of needle injury to make recommendations.

\subsection{Evaluation indicators}

In the course of the study, record the values of the survey scores of the surgical nurses, as well as the interviews with the interviews, and form the table data for observation and analysis.

\section{4 data processing}

The data of this study were SPSS 16.0 software for data Distributed under creative commons license 4.0 Volume 1; Issue 2 
entry, using descriptive statistical methods.

\section{Results}

2.1 Needle injury occurred
In the survey data show that 140 of the staff surveyed, the emergence of needle injury care workers a total of 87 , accounting for $62.1 \%$ of the total number.

\subsection{The emergence of needle injury work situation}

Table 1 Appears the work of needle injury

\begin{tabular}{ccc}
\hline Work links & Number of cases (n) & Percentage (\%) \\
\hline Extraction of syrup & 16 & 18.4 \\
Intravenous dosing & 15 & 17.2 \\
Needle removal & 21 & 24.1 \\
Draw off blood & 6 & 6.9 \\
When the syringe is discarded & 26 & 30 \\
Other & 3 & 3.4 \\
Total & 87 & 100 \\
\hline
\end{tabular}

\subsection{After the treatment of needle injury situation}

Table 2 After the treatment of needle injury situation

\begin{tabular}{ccc}
\hline Work links & Number of cases (n) & Percentage (\%) \\
\hline $\begin{array}{c}\text { Wound sterilization afeter wound } \\
\text { extrusion }\end{array}$ & 17 & 19.6 \\
$\begin{array}{c}\text { Wound squeezed out blood then syrup } \\
\text { rinse }\end{array}$ & 25 & 28.7 \\
Wound squeezed out blood then water \\
$\quad$ rinse & 19 & 21.8 \\
$\quad$ Wound water rinse & 10 & 11.5 \\
Not taking action & 16 & 18.4 \\
Total & 87 & 100 \\
\hline
\end{tabular}

\subsection{Dealing with countermeasures}

1) To raise awareness: For the staff in our hospital for surgical care, we must regularly carry out occupational safety education, and enhance the awareness of self-protection of nurses to strengthen the understanding of needle injury, protection, and processing of the curriculum, for the new induction of staff. In the pre-job training, we must pay attention to the concept of new staff training, including needle injury and other sharp injuries will bring harm to cultivate new employee self-protection awareness and awareness of prevention,standardized surgical care the process, strictly abide by the process requirements ${ }^{[2]}$.

2) Needle Injury Correctly Handle Training: Needle at any time may cause harm to the physical safety of the caregiver. In order to reduce the possibility of infection by the nursing staff, it is necessary to carry out the training of the nursing staff after the injury. To the wound at the blood out, the use of syrup cleaning and disinfection, and then wound, after injury, the report to the hospital in time to carry out serum testing, the hospital should develop a perfect surgical procedures and strict surgical care, then follow the process to deal with the wound $d^{[3]}$.

\section{Discussion}

Surgical care staff in the care of patients facing of a variety of hazards, which is the highest incidence of needle injury. Especially when nurses and patients have more contact in the intravenous or blood drawing, it is prone to needle injury, resulting in a certain probability of being transmitted to some blood-borne diseases. China is a high incidence of hepatitis B countries. In the treatment, many nurses are infected, therefore, in order to protect the physical safety of nurses and improve the safety of nurses working environment for surgical care staff must be occupational safety awareness of the training to improve the need to prevent needle injury ${ }^{[4]}$.

In this study, we can see that the current surgical care workers in the work, the emergence of a greater risk of 
needle injury, needle injury care workers were 87 , the total number of $62.1 \%$, which led to care personnel in the work of needle injury. When the syringe is abandoned, the patient for intravenous injection and needle is the highest frequency, which also shows that surgical care workers in the studio, especially syringes and other waste treatment are more casual, not strictly in accordance with the standards to implement. The staff of the wound treatment is not standardized. In the emergence of needle injury surgical care workers, improper handling of surgical injuries after the number of injuries accounted for $51.7 \%$ of the number, which has more than half. Nurses should pay attention to safety precautions, to strengthen the treatment after an injury.

\section{References}

[1] Yan Lihua, Zhao Xue, Bai Qiaofeng. Surgical Care Workers in the Work of Needle Injury Causes and Countermeasures. Contemporary nurses, 2016, 7(1): 25-26.

[2] Fu Yuezhen. Nursing Staff in the Work of Needle Injury Analysis and Countermeasures. Chinese Journal of Contemporary Medicine, 2013, 10(12): 168-169.

[3] Wei Lumei. Department of Nurses Needle Injury Occurred in the Analysis and Countermeasures. Chinese National Folk Medicine, 2013, 16(21): 144-146. 